Recebido em 10/2015. Aceito para publicação em 02/2016.

\title{
PREVALÊNCIA DE DOENÇAS CONCOMITANTES E COMPLICAÇÕES EM HIPERTENSOS DE UMA ÁREA DA ESTRATÉGIA "SAÚDE DA FAMÍLIA" DE CAXIAS-MA
}

\author{
CONCOMITANT DISEASE PREVALENCE AND COMPLICATIONS IN \\ HYPERTENSIVE OF AN AREA OF "HEALTH FAMILY STRATEGY" OF CAXIAS-MA \\ Wlyanna Araújo Abreu ${ }^{1}$ \\ Nytale Lindsay Cardoso Portela ${ }^{2}$
}

\begin{abstract}
Resumo: A hipertensão arterial sistêmica é uma doença silenciosa, diagnosticada muitas vezes no aparecimento das complicações, causando significativa perda na qualidade de vida e aumento nas taxas de morbidade e mortalidade. Objetiva-se identificar a presença de doenças concomitantes e complicações associadas ao processo hipertensivo em portadores de hipertensão arterial sistêmica assistidos por uma Estratégia Saúde da Familia de Caxias-MA. Trata-se de uma pesquisa quantitativa, realizada com 200 hipertensos. A coleta de dados se deu por um questionário estruturado durante os meses de janeiro e fevereiro de 2015, sendo a análise feita com a utilização do software Statistical Package for the Social Sciences. Constatou-se que 127 (63,5\%) entrevistados são mulheres e 73 (36,5\%) são homens. A idade variou de 30 anos ou mais, prevalecendo a faixa etária de 70 a 79 anos, com $48(24,0 \%)$ indivíduos. Em relação ao nível de escolaridade, predominou o ensino fundamental incompleto, seguido de não sabe ler/escrever. Quanto a presença de doenças concomitantes, constata-se que 128 (64,0\%) entrevistados não as possuem. Dos 72 indivíduos que apresentaram alguma doença concomitante, 44 (61,1\%) possuem diabetes tipo 1 ou 2. Em relação à presença de complicações, evidenciou-se que 139 (69,5\%) pacientes não apresentaram complicação. Das complicações apresentadas, os problemas de visão foram citados em maior número, com $22(36,1 \%)$ casos. Apesar de apenas uma pequena parcela da população apresentar doenças concomitantes e complicações, é imprescindivel a conscientização acerca da necessidade de se modificar o estilo de vida, praticando atividades físicas regularmente e mantendo dieta equilibrada para a prevenção de futuras complicações.
\end{abstract}

Palavras-chave: hipertensão; complicações; prevenção de doenças.

Abstract: Systemic arterial hypertension is a silent disease, often diagnosed in the appearance of complications, causing significant loss in quality of life and increased morbidity and mortality rates. The objective is to identify the presence of concomitant diseases and complications associated with hypertensive process in systemic arterial hypertension sufferers assisted by a Health family Strategy of Caxias-MA. It is a quantitative survey of 200 hypertensive persons. The data collection it was given by a structured questionnaire during the months of January and February 2015, being the analysis using the Statistical Package for Social Sciences software. It was found that 127 (63.5\%) respondents are female and 73 (36.5\%) are men. Ages ranged from 30 years or more, whichever age group 70-79 years, with 48 (24.0\%) individuals. Regarding the level of education, dominated the elementary school, followed by can not read / write. As the presence of concomitant diseases, it appears that $128(64.0 \%)$ respondents did not have them. Of the 72 individuals who had some concomitant disease, 44 (61.1\%) have diabetes type 1 or 2. In relation to the presence of complications, it became clear that 139 (69.5\%) patients had no complications. The presented complications, eyesight problems were cited in greater numbers with 22 (36.1\%) cases. Although only a small portion of the population present concomitant diseases and complications, the awareness of the need to modify the lifestyle is essential, practicing regular physical activity and maintaining a balanced diet to prevent future complications.

\footnotetext{
${ }^{1}$ Graduada em Enfermagem. Pós-graduanda em Urgência, Emergência e Atendimento Pré-hospitalar Universidade Estadual do Maranhão - UEMA, Brasil. E-mail: wlyannaacd@hotmail.com.

${ }^{2}$ Graduada em Enfermagem pelo Centro de Estudos Superiores de Caxias, da Universidade Estadual do Maranhão - CESC/UEMA, Brasil. Mestranda em Epidemiologia em Saúde Pública pela Fiocruz, Brasil. E-mail: nytalelindsay@hotmail.com.
} 
Keywords: hypertension; complications; disease prevention.

\section{INTRODUÇÃO}

A pressão arterial, conhecida também como tensão arterial, é a pressão exercida pelo sangue contra a superfície das artérias (NOBRE et al., 2013). Seus valores alteram quando há mudança na resistência periférica ou no débito cardíaco, podendo ser encontrada com um valor inferior a 120/80 mmHg (PA normal), de 120 a $129 / 80 \mathrm{mmHg}$ (pré-hipertensão) e de $140 / 90 \mathrm{mmHg}$ ou mais (hipertensão) (SMELTZER; BARE, 2011).

Estudos populacionais que avaliam a prevalência da hipertensão arterial no Brasil sobre a real situação da doença no país como um todo são ainda desconhecidos (NOBRE et al., 2013). Conforme Brasil (2006), a prevalência da hipertensão na população urbana adulta brasileira varia de 22,3\% a 43,9\% (média de $32,5 \%$ ). De acordo com a idade, observa-se que mais de $50 \%$ encontram-se na faixa etária compreendida entre 60 e 69 anos e 75\% acima de 70 anos, e entre os gêneros a prevalência foi de $35,8 \%$ nos homens e de $30 \%$ em mulheres.

A hipertensão arterial sistêmica (HAS) é uma doença silenciosa, inicialmente sem sintomas, e diagnosticada, muitas vezes, no aparecimento das complicações e, comumente, nas unidades de emergência, tardiamente, causando significativa perda na qualidade de vida e aumento nas taxas de morbidade e mortalidade (SOCIEDADE..., 2010). Com isso, torna-se importante a aferição anual da pressão arterial.

Associa-se, frequentemente, a alterações funcionais e/ou estruturais dos órgãosalvo (coração, encéfalo, rins e vasos sanguíneos) e a alterações metabólicas, com consequente aumento do risco de eventos cardiovasculares fatais e não fatais (MENEZES; GOBBI, 2013).

O surgimento da HAS está intimamente relacionado a fatores genéticos, ambientais e sociais. Dentre eles, destacam-se a idade, gênero e etnia, fatores socioeconômicos, excesso de peso e obesidade, sedentarismo, ingestão de álcool, tabagismo e hábitos alimentares, como a alta ingestão de sódio (MAGRINI; MARTINI, 2012). Além desses, a hereditariedade, raça, estresse e uso de anticoncepcionais.

O conhecimento dos fatores de risco torna-se essencial devido a HAS ser o principal fator de risco para as complicações mais comuns, entre elas, o desenvolvimento de doença arterial coronariana, acidente vascular encefálico, doença vascular periférica, insuficiência renal crônica, insuficiência cardíaca congestiva e infarto agudo do miocárdio (RUFINO et al., 2012).

Com a finalidade de diminuir os impactos decorrentes da HAS, o Ministério da Saúde desenvolveu o Plano de Reorganização da Atenção à HAS e ao diabetes melito (DM), no ano de 2002, com o objetivo primordial de acompanhar os hipertensos e 
diabéticos. Com ele, iniciou-se a inscrição nacional desses indivíduos no Sistema de Cadastramento e Acompanhamento de Hipertensos e Diabéticos (HiperDia) e no Programa Nacional de Assistência Farmacêutica para a HAS e o DM (LIMA et al., 2011).

O Plano de Reorganização da Atenção à HAS e ao DM é considerado uma das maiores propostas de intervenção para indivíduos com essas doenças, bem como os fatores de risco das doenças cardiovasculares. Esse programa tem como objetivo reverter esse cenário, estabelecendo metas e diretrizes para ampliar ações de prevenção, diagnóstico, tratamento e controle dessas patologias, por intermédio da reorganização do trabalho de atenção à saúde, das unidades da rede básica dos Serviços de Saúde (LIMA et al., 2012).

Entretanto, para que as ações estipuladas nesse plano sejam efetivas, é necessário que elas acompanhem as transformações da demanda atendida e essa informação é obtida pela base de dados do sistema HiperDia e do desenvolvimento e publicação de estudos que descrevam as características da população de diabéticos e hipertensos (LIMA et al., 2011).

Mesmo com a criação de políticas e/ou programas para a prevenção das doenças cardiovasculares, ressalta-se que esta pode não ser garantia de resolutividade do problema. Para isso, faz-se necessária a adesão do usuário ao esquema terapêutico proposto. Tal adesão depende de três fatores: do indivíduo estar ciente de sua condição de saúde e comprometido com o tratamento, dos profissionais de saúde através dos esclarecimentos e incentivos e do apoio familiar (CARVALHO et al., 2012).

Assim, o objetivo do trabalho foi identificar a presença de doenças concomitantes e complicações associadas ao processo hipertensivo em portadores de hipertensão arterial sistêmica, assistidos por uma Estratégia Saúde da Família de Caxias-MA.

\section{MATERIAL E MÉTODOS}

Trata-se de uma pesquisa com abordagem quantitativa e caráter descritivo, realizada em uma unidade básica de saúde (UBS) do município de Caxias-MA, escolhida por meio de sorteio. A amostra foi constituída por usuários dessa unidade de saúde, que preencheram os seguintes critérios de inclusão: estar cadastrado na UBS, no mínimo, há seis meses; ser hipertenso com diagnóstico comprovado; morar na área de abrangência da ESF selecionada; e aceitar participar da pesquisa mediante assinatura do Termo de Consentimento Livre e Esclarecido (TCLE). 
Foram excluídos aqueles pacientes que apresentaram dificuldade para responder às perguntas e estavam desacompanhados e os que se recusaram a participar do estudo.

Para o cálculo do tamanho da amostra, utilizou-se a fórmula de Barbetta (2006). A amostra $(n=200)$ permitiu estimar o parâmetro com margem de erro tolerável de $3 \%$ e nível de confiança de 97\%, na população finita de 244 pacientes hipertensos assistidos pela UBS sorteada.

Para a coleta de dados, foi utilizado, como instrumento, um questionário estruturado, desenvolvido pelas pesquisadoras, com base em informações da literatura. Para a aplicação do questionário, foram feitas visitas domiciliares, juntamente com os agentes comunitários de saúde da Unidade.

Os dados foram coletados nos meses de janeiro e fevereiro de 2015, nos turnos matutino e vespertino. Depois de preenchidos, os dados foram digitados, revisados e processados pela própria pesquisadora, sendo analisados e interpretados, com a utilização do software Statistical Package for the Social Sciences (SPSS, versão 20.0 for Windows), em um contexto quantitativo, expressos mediante símbolos numéricos. A análise estatística foi descritiva, sendo organizada em forma de tabelas e figuras para análise e discussão.

O estudo foi iniciado mediante o consentimento da Coordenação da Atenção Básica, aprovação da realização da pesquisa pelo Comitê de Ética em Pesquisa (CEP) do Centro de Estudos Superiores de Caxias, da Universidade Estadual do Maranhão (CESC/UEMA), sob o parecer no 912.371/2014 e CAAE no 37796314.7.0000.5554, e aceite dos sujeitos de participar, voluntariamente, da pesquisa, por intermédio da apresentação do TCLE assinado.

\section{RESULTADOS E DISCUSSÃO}

\section{Características sociodemográficas}

Dos 200 portadores de HAS que responderam ao questionário, a idade variou de 30 anos ou mais, prevalecendo a faixa etária de 70 a 79 anos, com $48(24,0 \%)$ indivíduos, seguida da faixa etária de 50 a 59 anos, com 46 (23,0\%), 60 a 69 anos, com 39 (19,5\%), 40 a 49 anos, com 23 (11,5\%), 80 a 89 anos, com 21 (10,5\%), 30 a 39 anos, com $14(7,0 \%)$ e 90 ou mais anos, com $09(4,5 \%)$. Em relação ao sexo, constatou-se que 127 (63,5\%) são mulheres e 73 (36,5\%) são homens.

No que diz respeito a cor, observou-se que $128(64,0 \%)$ entrevistados se auto definiram como pardos, $50(25,0 \%)$ como negros, 17 (8,5\%) como brancos e $05(2,5 \%)$ 
como amarelos.

Quanto ao estado civil, constatou-se que $90(45,0 \%)$ investigados são casados. Os outros portadores de HAS são viúvos, 57 (28,5\%); solteiros, 20 (10,0\%); vivem em união estável, 18 (9,0\%); e separados, 15 (7,5\%).

Em relação ao nível de escolaridade, predominou o ensino fundamental incompleto com 74 (37\%) casos, seguido de não sabe ler/escrever com 63 (31,5\%), fundamental completo com $28(14,0 \%)$, médio completo com $24(12,0 \%)$, superior completo com $04(2,0 \%)$, médio incompleto com 03 (1,5\%), superior incompleto com $03(1,5 \%)$ e especialização/residência com 01 (0,5\%).

Com relação à renda familiar, a tabela evidenciou que $132(66,0 \%)$ participantes se mantêm com mais de um salário mínimo, enquanto que 68 (34,0\%) têm uma renda menor que um salário mínimo.

\section{Histórico de doenças concomitantes}

Na figura 1, constata-se que $128(64,0 \%)$ entrevistados não possuem doença associada à HAS. Esses dados diferem-se da pesquisa de Ramos (2013), desenvolvida no município de Caxias-MA, com 356 hipertensos de mais de 60 anos cadastrados no programa HiperDia, na qual 72 (76,9\%) informaram ter outros problemas de saúde.

Figura 1. Número e distribuição percentual dos portadores de Hipertensão Arterial Sistêmica assistidos por uma Estratégia Saúde da Família, de acordo com a presença de doenças concomitantes, Caxias, Maranhão, 2015.

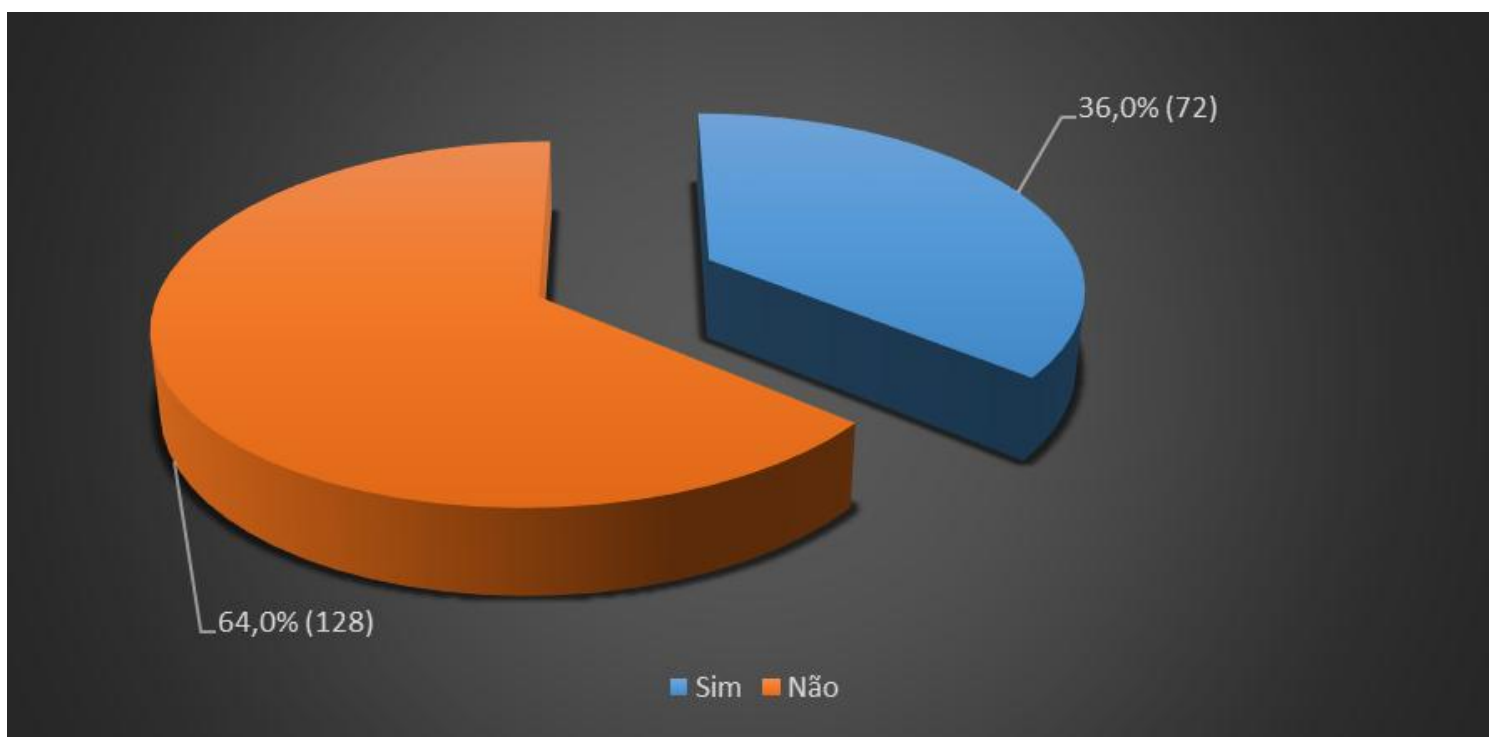

Fonte: Autora.

Girotto et al. (2013), ao realizarem estudo com 385 hipertensos de 20 a 79 anos, com o objetivo de determinar a adesão aos tratamentos farmacológico e não 
farmacológico da hipertensão arterial na atenção primária e identificar fatores associados, encontraram que 177 (46,0\%) hipertensos informaram não ter comorbidades associadas, o que difere do encontrado no presente estudo.

Conforme a Sociedade... (2010), o hipertenso com pressão arterial normal e possuindo três ou mais fatores de risco, ou lesão de órgãos alvo ou diabetes, já apresenta risco médio adicional para doença cardiovascular. Em pessoas com pressão arterial limítrofe, esse risco eleva-se para alto e, assim, sucessivamente.

Tabela 1. Número e distribuição percentual dos portadores de Hipertensão Arterial Sistêmica assistidos por uma Estratégia Saúde da Família, de acordo com o histórico de doenças concomitantes, Caxias, Maranhão, 2015.

\begin{tabular}{lc}
\hline \multicolumn{1}{c}{ HISTÓRICO DE DOENÇAS CONCOMITANTES } & $\mathbf{N}(\%)$ \\
\hline Diabetes melito Tipo 1 & $19(26,4)$ \\
Diabetes melito Tipo 2 & $25(34,7)$ \\
Outra(s) ${ }^{(1)}$ & $28(38,9)$ \\
\hline TOTAL & $72(100,0)$ \\
\hline
\end{tabular}

Nota: ${ }^{(1)}$ Dislipidemia, hipercolesterolemia, osteoporose.

Fonte: Autora.

$\mathrm{Na}$ tabela 1, verifica-se que 72 indivíduos dos 200 entrevistados apresentaram alguma doença concomitante, sendo que $44(61,1 \%)$ deles possuem diabetes tipo 1 ou 2 , e $28(38,9 \%)$ possuem outras doenças, tais como dislipidemia, hipercolesterolemia e osteoporose.

De acordo com Brandão et al. (2010), a possibilidade de associação da HAS e do diabetes melito (DM) é da ordem de $50 \%$, o que, não raro, requer o manejo das duas doenças no mesmo usuário, agravado pelo fato de que sua concomitância potencializa o dano micro e macro vascular decorrente, acarretando alta morbidade cardiocerebrovascular. Além disso, Silva et al. (2011) ressaltam que ambas são consideradas como um dos principais fatores de risco para as doenças cardiovasculares.

Santos e Moreira (2012), com o objetivo de identificar os fatores de risco e as complicações associadas em usuários com hipertensão/diabetes de Fortaleza-CE, identificaram que $660(29,4 \%)$ indivíduos tem HAS associada a DM. Silva et al. (2011) verificaram em seu estudo que 58 (19,5\%) hipertensos, possuíam hipertensão associada ao diabetes, sendo um fator de risco adicional relevante para essa clientela, principalmente para os idosos, que já sofrem alterações no organismo devido o envelhecimento.

Segundo Girotto et al. (2013) e Duarte et al. (2013), indivíduos com HAS e doenças associadas têm necessidade de vários medicamentos para manter as patologias sobre controle e, dessa forma, oneram o orçamento individual e familiar, além de dificultar os cuidados com os horários e dosagens. 
Sabe-se que quanto maior a idade, mais difícil o controle da pressão arterial, pois a própria senescência leva a uma limitação fisiológica que poderá intervir na adesão ao tratamento de forma correta. Diante disso, observou-se, neste estudo, que a população é predominantemente adulta e idosa, destacando-se a relevância da conscientização dos malefícios da hipertensão arterial para essa parcela da população, objetivando que o indivíduo se torne elemento ativo no processo de tratar, assumindo o cuidado com sua saúde, e evitando que apresente doenças concomitantes.

Portanto, isso propõe uma reflexão sobre a qualidade de vida desses hipertensos atendidos na unidade de saúde investigada, na adoção de medidas individuais ou coletivas que visem a mudanças de hábitos nocivos à saúde para práticas saudáveis e regulares, com o objetivo de controlar a hipertensão e melhorar a expectativa de vida, principalmente, por aqueles que já possuem outras doenças associadas.

\section{Complicações}

No presente estudo, evidenciou-se que 139 (69,5\%) dos pacientes hipertensos não apresentaram complicação (FIGURA 2). Valores mais elevados foram encontrados por Sousa et al. (2014), em uma pesquisa realizada com usuários do Programa Hiperdia, em Jequié-BA, revelando que 3.307 (72,7\%) entrevistados já apresentaram ou apresentam alguma complicação em decorrência da HAS.

Figura 2. Número e distribuição percentual dos portadores de Hipertensão Arterial Sistêmica assistidos por uma Estratégia Saúde da Família, de acordo com a presença de complicações, Caxias, Maranhão, 2015.

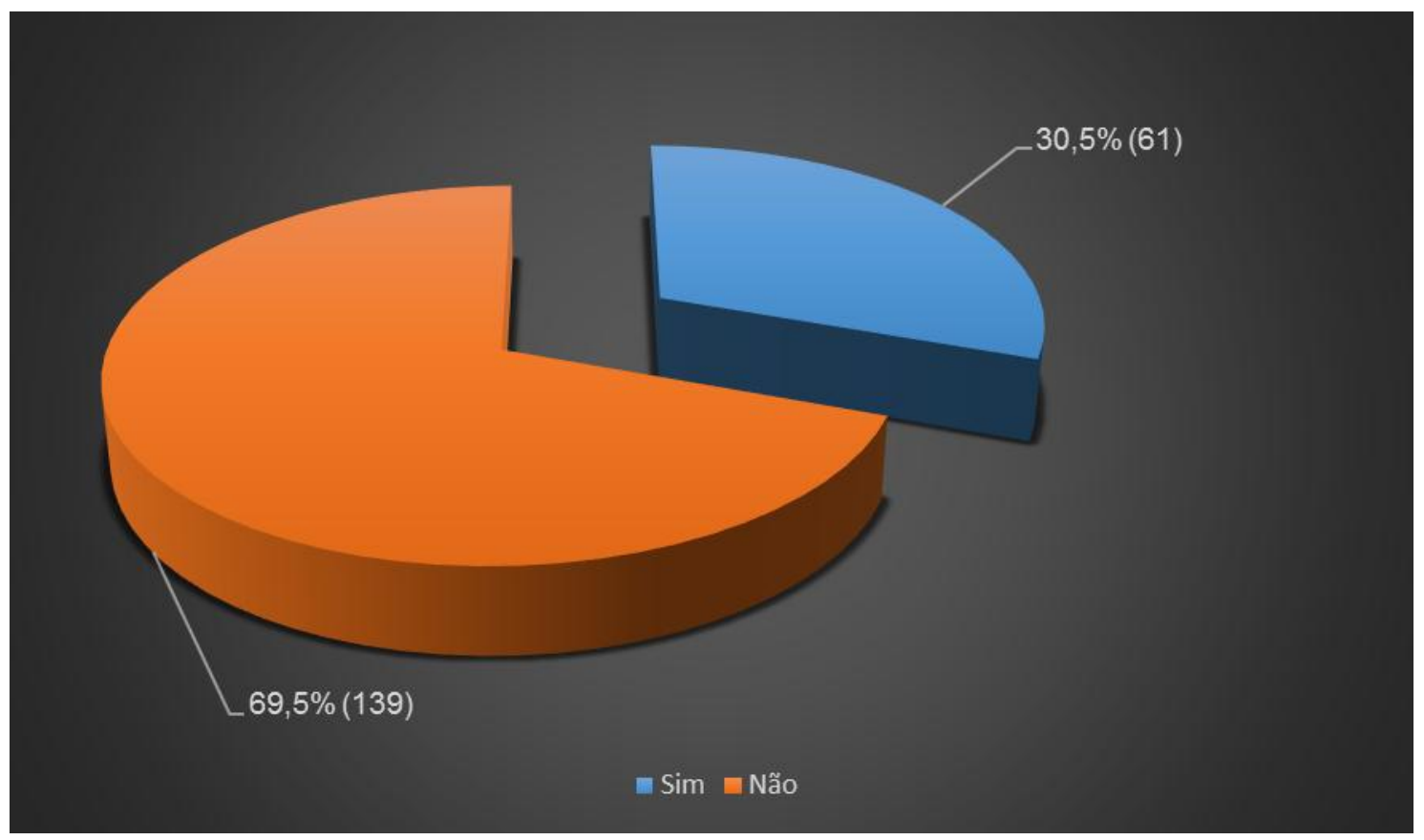

Fonte: Autora. 
Segundo Zattar et al. (2013), os idosos constituem um importante grupo de risco para os eventos catastróficos e, muitas vezes, incapacitantes relacionados à HAS, tais como doença arterial coronariana, doença cerebrovascular doença vascular periférica, insuficiência cardíaca, doença renal terminal e outras complicações. Ainda sobre o autor, a dependência funcional de moderada a grave, imposta pelo envelhecimento, está associada a maior prevalência de pressão arterial elevada, sendo esta a condição crônica que mais frequentemente influencia a capacidade funcional de idosos.

Tabela 2. Número e distribuição percentual dos portadores de Hipertensão Arterial Sistêmica assistidos por uma Estratégia Saúde da Família, de acordo com a presença de complicações, Caxias, Maranhão, 2015.

\begin{tabular}{lc}
\hline \multicolumn{1}{c}{ PRESENÇA DE COMPLICAÇÕES } & $\mathbf{N}(\%)$ \\
\hline Problemas de visão & $22(36,1)$ \\
Acidente Vascular Encefálico (AVE) & $17(27,9)$ \\
Infarto Agudo do Miocárdio (IAM) & $10(16,4)$ \\
Doenças renais & $09(14,7)$ \\
Outra(s) $^{(1)}$ & $03(4,9)$ \\
\hline TOTAL & $61(100,0)$ \\
\hline
\end{tabular}

Fonte: Autora.

Quando observamos as complicações apontadas na Tabela 2, percebemos que os problemas de visão foram citados em maior número, com $22(36,1 \%)$ casos, seguido de acidente vascular encefálico, com 17 (27,9\%), infarto agudo do miocárdio, com $10(16,4 \%)$ e doenças renais, com 09 (14,7\%). Pode-se constatar, ainda, que 03 $(4,9 \%)$ usuários afirmaram ter doença coronária.

Esses dados encontrados corroboram com os da pesquisa de Sousa et al. (2014), realizada na cidade Jequié-BA, com usuários do Programa HiperDia, na qual identificou-se que $519(4,37 \%)$ sujeitos tiveram complicação relacionada a problemas de visão; 456 (3,84\%), o acidente vascular cerebral; o infarto agudo do miocárdio apresentou $306(2,57 \%)$ casos e as doenças renais tiveram $256(2,15 \%)$.

Os problemas relacionados à visão são apontados como a principal complicação que acomete esses usuários. Em um estudo desenvolvido por Ferreira et al. (2011), no qual buscou-se avaliar as condições de saúde dos idosos residentes na zona urbana do município de Uberaba-MG, observaram que os problemas cardíacos e de visão estão relacionados com o declínio funcional e com o tipo de morbidade, devido à incapacidade funcional imposta pelo envelhecimento.

Para Zattar et al. (2013), o acidente vascular encefálico, que tem a hipertensão arterial sistêmica como o principal fator de risco modificável, é uma das principais causas de dependência funcional para idosos, e tem o potencial de levar a complicações, como incontinência urinária, disfagia, depressão e dor crônica.

Girotto et al. (2013) constataram a prevalência do IAM na população estudada como uma das complicações da HAS. Segundo esses autores, indivíduos que 
apresentam outras comorbidades, além da hipertensão, podem receber maior atenção dos profissionais e serviços de saúde, o que pode resultar em melhora nos índices de adesão ao tratamento farmacológico.

A pressão arterial não controlada representa um risco significativo de eventos cardiocerebrovasculares e renais, o que pode prejudicar a sua qualidade de vida e, até mesmo, levar à morte, sendo, portanto, necessárias ações para evitar tais desfechos.

\section{CONCLUSÃO}

Diante dos resultados obtidos, ressalta-se a necessidade de os pacientes hipertensos receberem orientação quanto à importância do uso dos medicamentos de forma correta, dos fatores de risco associados à doença, cronicidade da doença, ausência de sintomatologia específica e complicações que comprometem órgãos vitais quando não controlados os níveis tensionais, para que evitem as doenças associadas e as complicações decorrentes da HAS.

Somando a esses fatores, é imprescindível a conscientização acerca da necessidade de se modificar o estilo de vida, praticando atividades físicas regularmente e mantendo dieta equilibrada para a prevenção de futuras complicações. Somente com a orientação e conscientização dos portadores de hipertensão arterial sistêmica que se pode evitar complicações.

Ressalta-se, portanto, a importância de os profissionais de saúde criarem vínculos com os pacientes, estabelecendo um diálogo proveitoso com uma linguagem compreensível e tratamento individualizado, para que seja possível fazer que os indivíduos mudem suas atitudes e valores.

\section{REFERÊNCIAS}

BARBETTA, P. A. Estatística aplicada às ciências sociais. 6. ed. Florianópolis: UFSC, 2006.

BRANDÃO A. et al. VI Diretrizes Brasileiras de Hipertensão Arterial. Arquivos Brasileiros de Cardiologia, v. 95, 1 Supl 1, p. I-III, 2010.

BRASIL. Ministério da Saúde. Secretaria de Atenção à Saúde. Departamento de Atenção Básica. Hipertensão arterial sistêmica para o Sistema Único de Saúde. Brasília: Ministério da Saúde, 2006.

CARVALHO, A. L. M. et al. Adesão ao tratamento medicamentoso em usuários cadastrados no Programa Hiperdia no município de Teresina (PI). Ciência \& Saúde coletiva [online], v. 17, n. 7, p. 1885-1892, 2012. Disponível em:

<http://dx.doi.org/10.1590/S1413-81232012000700028>. Acesso em: 17 abril 2015. 
DUARTE, O. et al. Tratamento Ambulatorial da Hipertensão Arterial Sistêmica revisão de literatura. Revista UNINGÁ Review, v.17, n.2, p.22-29, 2013.

FERREIRA, P. C. S. et al. Características sociodemográficas, capacidade funcional e morbidades entre idosos com e sem declínio cognitivo. Acta Paulista de Enfermagem, v. 24, n. 1, p. 29-35, 2011.

GIROTTO, E. et al. Adesão ao tratamento farmacológico e não farmacológico e fatores associados na atenção primária da hipertensão arterial. Ciênc. Saúde Coletiva [online], v. 18, n. 6, p. 1763-1772, 2013.

LIMA, A. S. et al. A importância do Programa Hiperdia em uma Unidade de Saúde da Família do município de Serra Talhada - PE, para adesão dos hipertensos e diabéticos ao tratamento medicamentoso e dietético. Saúde Coletiva em Debate, v. 2, n. 1, p. 917, dez. 2012.

LIMA, L. M. et al. Perfil dos usuários do Hiperdia de três unidades básicas de saúde do sul do Brasil. Revista Gaúcha de Enfermagem [online], v. 32, n. 2, p. 323-329, 2011. Disponível em: <http://dx.doi.org/10.1590/S1983-14472011000200016>. Acesso em: 17 abril 2015.

MAGRINI, D. W.; MARTINI, J. G. Hipertensão arterial: principais fatores de risco modificáveis na estratégia saúde da família. Enfermería Global, n. 26, p. 354-363, 2012.

MENEZES, A. G. M. P.; GOBBI, D. Educação em saúde e Programa de Saúde da Família: Atuação da enfermagem na prevenção de complicações em pacientes hipertensos. O Mundo da Saúde, v. 34, n. 1, 2013.

NOBRE, F. et al. Hipertensão Arterial Sistêmica Primária. Medicina, v. 46, n. 3, p. 256272, 2013.

RAMOS, J. S. Avaliação da Adesão ao Tratamento por Idosos cadastrados no Programa Do Hiperdia. Caxias (MA). Monografia. Universidade Estadual do Maranhão, Centro de Estudos Superiores de Caxias, 2013.

RUFINO, D. B. R. et al. Adesão ao tratamento: estudo entre portadores de hipertensão arterial cadastrados em uma Unidade Básica de Saúde. Journal of the Health Sciences Institute, v. 30, n. 4, p. 336-342, 2012.

SANTOS, J. C.; MOREIRA, T. M. M. Fatores de risco e complicações em hipertensos/diabéticos de uma regional sanitária do nordeste brasileiro. Revista da Escola de Enfermagem da USP, v. 46, n. 5, p. 1125-1132, 2012.

SOCIEDADE BRASILEIRA DE CARDIOLOGIA. Sociedade Brasileira de Hipertensão. Sociedade Brasileira de Nefrologia. VI Diretrizes Brasileiras de Hipertensão. Arquivos Brasileiros de Cardiologia, v.17, n.1, 2010.

SILVA, D. B. et al. Associação entre hipertensão arterial e diabetes em centro de saúde da família. RBPS, v. 24, n. 1, p. 16-23, 2011.

SMELTZER, S. C.; BARE, B. G. Brunner \& Suddarth: Tratado de Enfermagem Médico-Cirúrgica. 12 ed. Rio de Janeiro: Guanabara, 2011.

SOUZA, S. S. et al. Análise do perfil da hipertensão e diabetes no município de JequiéBa. InterScientia, v.2, n.1, p. 63-76, jan./abr. 2014. 
ZATTAR, L. C. et al. Prevalência e fatores associados à pressão arterial elevada, seu conhecimento e tratamento em idosos no sul do Brasil. Cadernos de Saúde Pública, v. 29, n. 3, p. 507-521, 2013. 